\title{
MCNP-X Monte Carlo Code Application for Mass Attenuation Coefficients of Concrete at Different Energies by Modeling $3 \times 3$ Inch NaI(Tl) Detector and Comparison with XCOM and Monte Carlo Data
}

\author{
Huseyin Ozan Tekin \\ Vocational School of Health Service, Radiotherapy Department, Uskudar University, 34672 Istanbul, Turkey \\ Correspondence should be addressed to Huseyin Ozan Tekin; huseyinozan.tekin@uskudar.edu.tr \\ Received 1 May 2016; Revised 22 June 2016; Accepted 10 July 2016 \\ Academic Editor: Keith E. Holbert
}

Copyright (C) 2016 Huseyin Ozan Tekin. This is an open access article distributed under the Creative Commons Attribution License, which permits unrestricted use, distribution, and reproduction in any medium, provided the original work is properly cited.

\begin{abstract}
Gamma-ray measurements in various research fields require efficient detectors. One of these research fields is mass attenuation coefficients of different materials. Apart from experimental studies, the Monte Carlo (MC) method has become one of the most popular tools in detector studies. An $\mathrm{NaI}(\mathrm{Tl})$ detector has been modeled, and, for a validation study of the modeled $\mathrm{NaI}(\mathrm{Tl})$ detector, the absolute efficiency of $3 \times 3$ inch cylindrical $\mathrm{NaI}(\mathrm{Tl})$ detector has been calculated by using the general purpose Monte Carlo code MCNP-X (version 2.4.0) and compared with previous studies in literature in the range of 661-2620 keV. In the present work, the applicability of MCNP-X Monte Carlo code for mass attenuation of concrete sample material as building material at photon energies $59.5 \mathrm{keV}, 80 \mathrm{keV}, 356 \mathrm{keV}, 661.6 \mathrm{keV}, 1173.2 \mathrm{keV}$, and $1332.5 \mathrm{keV}$ has been tested by using validated NaI(Tl) detector. The mass attenuation coefficients of concrete sample have been calculated. The calculated results agreed well with experimental and some other theoretical results. The results specify that this process can be followed to determine the data on the attenuation of gammarays with other required energies in other materials or in new complex materials. It can be concluded that data from Monte Carlo is a strong tool not only for efficiency studies but also for mass attenuation coefficients calculations.
\end{abstract}

\section{Introduction}

Since radioactive sources have been extensively used in many fields such as medicine, industry, and energy applications, radiation detectors have played a major role in detection and quantification of ionizing radiations as well as radiation protection procedures. Among all types of radiation detectors, because of their higher detection efficiencies of gamma-rays, scintillation-based $\mathrm{NaI}(\mathrm{Tl})$ detectors have been used widely for radiation spectroscopy and radioisotopebased applications including medical and industrial areas [1]. Furthermore, these types of detectors have shown reliable results for low level radioactive source measurements because of higher detection efficiency and suitable features for operation at room temperature. However, the accuracy of measurement depends strongly on some detection properties of scintillation detectors such as detection efficiency and geometric efficiency. Experimental gamma spectrometry is an effective method to evaluate the efficiency of $\mathrm{NaI}(\mathrm{Tl})$ detectors. On the other hand, in recent years, the Monte Carlo (MC) method has been widely utilized for efficiency determination studies [2-5]. Absolute efficiency is defined as the ratio of the number of counts produced by the detector to the number of gamma-rays emitted by the source (in all directions). Some experimental and calculation studies have reported on detection efficiency determination [6-10]. Nowadays, radiation technology is starting to be used in a variety of different areas such as nuclear power bases, particle accelerators such as linac and synchrotron, and medical facilities such as nuclear medicine and radiological facilities and thus radiation protection becomes important. Shielding is widely and effectively used method for protection from radiation hazards; improvement of the shielding properties of concrete which is a commonly used construction material becomes important. These studies can be performed using 
efficient detectors. This study presents the use of $\mathrm{NaI}(\mathrm{Tl})$ detectors modeled by Monte Carlo method during mass attenuation coefficients calculations. The validity of the modeling geometry in Monte Carlo studies is critically important. Based on this reason, first step of this study was to create a geometry by using MCNP-X and afterwards a validity check to compare the efficiency results of the detector with available previous works in literature. The validated detector is used for mass attenuation coefficients calculations of simple concrete sample at $59.5 \mathrm{keV}\left({ }^{241} \mathrm{Am}\right), 80.9 \mathrm{keV}\left({ }^{133} \mathrm{Ba}\right)$, $140.5 \mathrm{keV}\left({ }^{99 \mathrm{~m}} \mathrm{Tc}\right), 356.5 \mathrm{keV}\left({ }^{133} \mathrm{Ba}\right), 661.6 \mathrm{keV}\left({ }^{137} \mathrm{Cs}\right), 1173.2$ $\left({ }^{60} \mathrm{Co}\right)$, and $1332.5 \mathrm{keV}\left({ }^{60} \mathrm{Co}\right)$ photon energies which are the most commonly used isotopes in nuclear investigations.

\section{Material and Methods}

2.1. Validation of Monte Carlo Model: Efficiency Calculations of Modeled NaI(Tl) Detector. Absolute efficiency must be known especially in radioactivity measurements. In this study, absolute efficiency of modeled detector in a wide energy range is calculated. The definition of the absolute efficiency is shown in

$$
\varepsilon_{\mathrm{abs}}=\frac{N_{c}}{N_{s}} .
$$

Here in (1), $N_{c}$ defines the number of counts recorded by detector and $N_{s}$ defines the number of radiations emitted by the source (all directions such as isotropic source). In this study, MCNP-X (Monte Carlo N-Particle eXtended) version 2.4.0 has been used for geometry design and calculations. MCNP-X is a general purpose radiation transport code for modeling the interaction of radiation with materials. MCNP$\mathrm{X}$ is fully three-dimensional and it utilizes extended nuclear cross section libraries and uses physics models for particle types. MCNP-X is a suitable and strong code that has a capability for various studies. The MCNP-X capability of detecting efficiency and using different experimental and Monte Carlo studies has been studied by Akkurt et al. [14]. Also, using conditions of MCNP-X for dose distribution has been studied by Tekin and Kara [15]. The detector geometry was modeled according to Figure 1. And physical parameters of related detector shown in Figure 1 were taken from Canberra Company and the same detector parameters also used earlier in some experimental studies [16]. The detector response function was determined by means of pulse-height tally named F8 tally in the MCNP-X input file. This tally scores the energy distribution of pulses created in a detector by radiation. The net response is the spectra of pulses with heights proportional to the frequency of events in distinct energy bins.

All compounds and pure materials of detector were defined in the MC input file. These materials were aluminum with density of $2.7 \mathrm{~g} / \mathrm{cm}^{3}, \mathrm{MgO}$ with density of $3.58 \mathrm{~g} / \mathrm{cm}^{3}$, $\mathrm{NaI}(\mathrm{Tl})$ with density of $3.67 \mathrm{~g} / \mathrm{cm}^{3}$, and $\mathrm{SiO}_{2}$ with density of $2.648 \mathrm{~g} / \mathrm{cm}^{3}$. A screenshot of the modeled $\mathrm{NaI}(\mathrm{Tl})$ detector by using MCNP-X code is shown in Figure 1. Due to the simulation process in code, the total simulation geometry is seen in Figure 2, and, as it can be seen, there is one

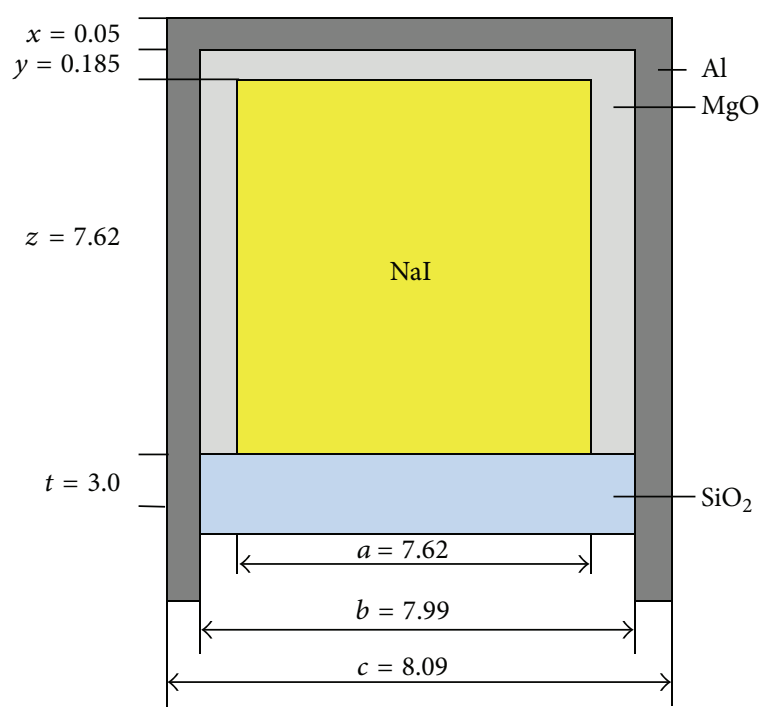

FIgURE 1: Schematic representation of $\mathrm{NaI}(\mathrm{Tl})$ detector considered in simulation.

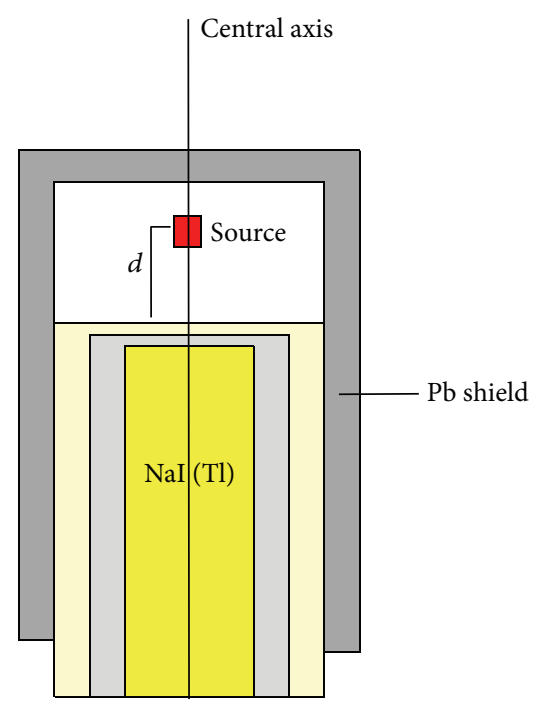

FIGURE 2: Considered source and detector location in simulation.

cylindrical $3 \times 3$ inch $\mathrm{NaI}(\mathrm{Tl})$ detector of height in crystal $7.62 \mathrm{~cm}$ and diameter $7.62 \mathrm{~cm}$ with a monoenergetic isotropic point source. Also, the source and detector assembly were shielded by lead blocks.

Simulation equipment such as detector and lead blocks have been defined in data cell card and surface card sections of MCNP-X input by considering different variables such as geometry, location, dimension, and density. The gamma-ray sources also have been defined in data card CEL, ERG, DIR, POS, and PAR. Each variable has different abilities during simulation. In present study, our variables commanded source cell, energy, direction, source position, and particle type, respectively. On the other hand, one of the important definitions is material specification by considering atomic number, mass number, and density for pure elemental materials and atomic number, mass number, elemental mass 
TABLE 1: Total efficiency values for $3 \times 3$ inch $\mathrm{NaI}(\mathrm{Tl})$ detector with a point source located $d=0.001 \mathrm{~cm}$ away from the front surface of the detector.

\begin{tabular}{lcccccc}
\hline \multirow{2}{*}{ Energy $(\mathrm{keV})$} & \multicolumn{5}{c}{ Total efficiency $(d=0,001 \mathrm{~cm})$} \\
& Present work & Yalcin et al. [11] & Vegors Jr. et al. [4] & Nakamura [12] & Miller and Snow [5] & MCNP-X error rate \\
\hline 661 & 0.3618 & 0.3646 & 0.362 & 0.367 & 0.370 & 0.0015 \\
1332 & 0.3011 & 0.2930 & 0.293 & 0.296 & 0.302 & 0.0016 \\
2620 & 0.2491 & 0.2476 & 0.248 & 0.249 & 0.250 & 0.0019 \\
\hline
\end{tabular}

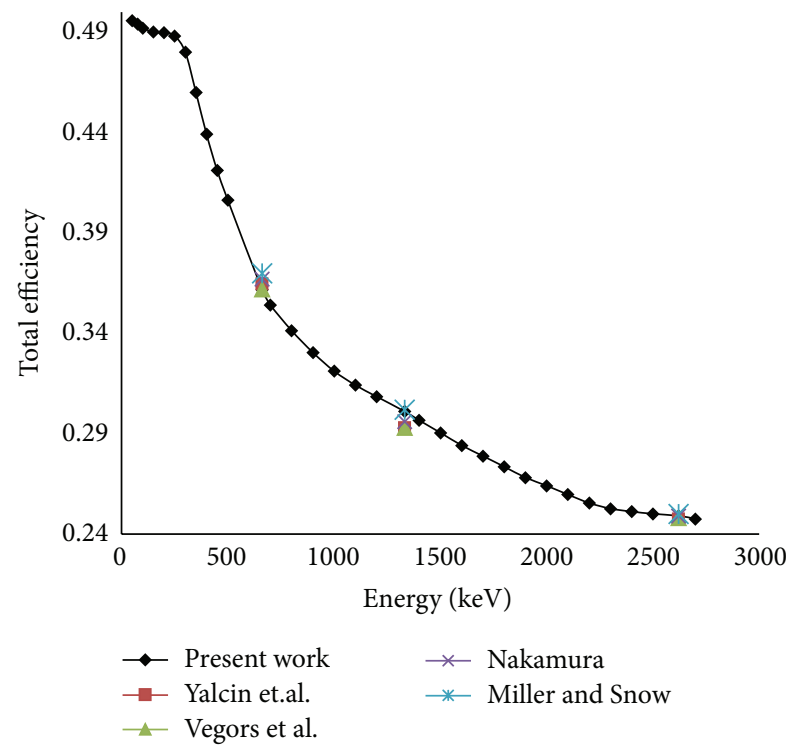

FIGURE 3: Total efficiency for $3 \times 3$ inch $\mathrm{NaI}(\mathrm{Tl})$ detector $(d=$ $0.001 \mathrm{~cm})$.

fraction, and density for compounds or mixtures. By considering these variables, material definitions of concrete have been done in simulation. When some energy $E$ is deposited into the detector, accounting the corresponding channel of the spectrum is recorded. Somehow, the gamma-rays spectra obtained in the simulations are very different from the spectra obtained with the detectors. Thus, the resolution calibration in the user code must be accounted for obtaining the realistic spectra [17]. As we know, the absolute efficiency of detector is dependent on the source-detector distance. In this study, simulations were repeated by considering distance of $d=$ $0.001 \mathrm{~cm}$ source-detector distance. The MC calculations for total efficiency were presented in Table 1, for $d=0.001 \mathrm{~cm}$ distance between source and detector. The geometric center of detector was considered for location of point source. $\mathrm{MC}$ calculations were done by using Intel ${ }^{\otimes} \mathrm{Core}^{\mathrm{TM}}$ i7 CPU $2.80 \mathrm{GHz}$ computer hardware. Also the comparisons were made between present study and other studies in these tables. The results show that with an increase in photon energy, the total efficiency is reduced for all distances in certain energy values. The reduced efficiency rates were given in Table 1. In this study, the absolute detector efficiency of a modeled $\mathrm{NaI}(\mathrm{Tl})$ detector for different energies of photons and for different source-detector distances was calculated by using Monte Carlo method with MCNP-X code. A good agreement was observed between the Monte Carlo and previous studies for $d=0.001 \mathrm{~cm}$ detector-source distance. Also in the simulation, isotropic point sources were considered for calculations. Total efficiency values were calculated for $3 \times 3$ inch $\mathrm{NaI}(\mathrm{Tl})$ detector for source-detector distances of $d=0.001 \mathrm{~cm}$. Since the Monte Carlo method has become an important tool in efficiency studies, MCNP-X code was used for this study successfully.

As we see from Figure 3, good agreement was achieved between efficiency values. Somehow, Hybrid Monte Carlo program which has been developed by Yalcin et al. [11] requires rather short computing time. In the present simulation, the run time was longer than in the Hybrid Monte Carlo method. In this study, good agreement is achieved between gamma energy and detector efficiency. As the gamma energy increases in interaction, the total detector efficiency decreases given that the possibility of a photon being absorbed inside of the detector decreases.

2.2. Mass Attenuation Coefficients Calculations. To avoid population and staff exposure to ionization radiation, working and public areas should be shielded. The main investigation required for such studies is mass attenuation coefficient values of building materials. Mass attenuation coefficient measures the probability of interaction of photon with the material. Modeling the photon attenuation through materials in a simulation environment gives more flexibility and simplicity of use and change of parameters instead of performing an experimental study of mass attenuation coefficients of different materials. Thus, modeling of validated detector geometry would be useful for future studies where the energy value should be changed. In this study, we carried out an investigation on availability of MCNP-X Monte Carlo code for calculation of mass attenuation coefficients of simple defined concrete used for mass attenuation coefficients calculations [13] The mass attenuation coefficient is one of the most important parameters for characterizing the penetration and diffusion of gamma-rays in any objective material [18]. Mass attenuation coefficients of investigated materials are determined by the transmission method according to BeerLambert's law:

$$
\mu_{m} \cdot x=\ln \left(\frac{I_{o}}{I}\right),
$$

where $I_{o}$ and $I$ are the incident and attenuated photon intensity, respectively, $\mu_{m}\left(\mathrm{~cm}^{2} \cdot \mathrm{g}^{-1}\right)$ is the mass attenuation coefficient, and $x$ is the thickness of the slab. In recent years, many researchers have studied determination of mass attenuation coefficients theoretically and experimentally for 
TABLE 2: Concrete sample parameters (density $\left.=2.3 \mathrm{~g} \cdot \mathrm{cm}^{-3}\right)$.

\begin{tabular}{lc}
\hline Element & Mass fraction (\%) \\
\hline $\mathrm{H}$ & 1 \\
$\mathrm{C}$ & 0.1 \\
$\mathrm{O}$ & 52.9107 \\
$\mathrm{Na}$ & 1.6 \\
$\mathrm{Mg}$ & 0.2 \\
$\mathrm{Al}$ & 3.3872 \\
$\mathrm{Si}$ & 33.7021 \\
$\mathrm{~K}$ & 1.3 \\
$\mathrm{Ca}$ & 4.4 \\
$\mathrm{Fe}$ & 1.4 \\
\hline
\end{tabular}

various materials, such as some experimental studies performed by Akkurt and El-Khayatt [19]. Effect of the boron carbide aluminum metal matrix composite on radiation shielding has been studied by Akkaş et al. [20]. Investigation of biological materials and their attenuations by comparing Monte Carlo and XCOM have been studied Medhat et al. [21]. Mass attenuation coefficients of composite material comparison have been studied by Medhat and Singh by using Geant4 and XCOM [22]. In this study, The mass attenuation coefficients of concrete sample defined in the simulation package were obtained by using the MCNP-X Monte Carlo code at 59.5, 80.9, 140.5, 356.5, 661.6, 1173.2, and $1332.5 \mathrm{keV}$ photon energies. Simple concrete material [23] content ratios have been defined as sample material in MCNP-X input. Elemental structure and mass fractions of used concrete are given in Table 2.

A complex elemental concrete has been defined in MCNP-X due to elemental structure and mass fraction in sample. However, the source was approximated as a point source with other equipment in simulation such as concrete sample and $\mathrm{NaI}(\mathrm{Tl})$ detector in lead $(\mathrm{Pb})$ shielding material. For a good interaction with $\mathrm{NaI}(\mathrm{Tl})$ detector, photons have been collimated onto detector the window in simulation. Since MCNP-X obtains the primary sources of nuclear data, evaluations from the evaluated nuclear data file (endf) system, evaluated nuclear data library (endl), and evaluated photon data library (epdl) are highly capable of photonic calculations. In this study, also some variance reduction techniques have been applied such as cutting off energy and reducing the types of observed particles in interaction such as ignoring of electrons in mother world and equipment geometries.

MC calculations were done by using Intel Core i7 CPU $2.80 \mathrm{GHz}$ computer and for one million starting particles per run (NPS). Depending on the long calculation run time, statistical error that has been obtained was less than $1 \%$. Of course, this error reduction not only depends on long run time but also depends on variance reduction methods such as cut-off energy applications in MCNP-X data card, ignoring the unused particles in simulation such as neutron and electron and optimized mother world volume in simulation geometry. The average cell flux tally (F4) has been used during mass attenuation coefficient calculation. This type of

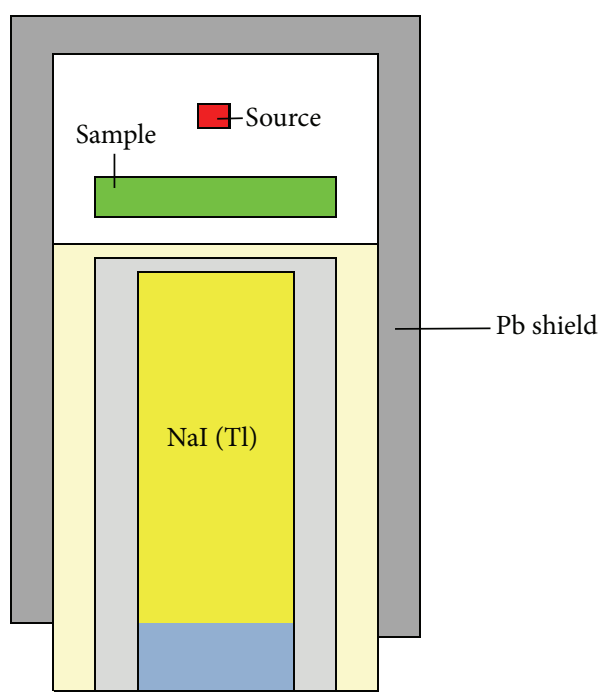

FIgURE 4: Schematic representation of simulation and locations of modeled equipment.

tally makes use of what may be called a variance reduction technique, namely, use of the next event estimator. For each source particle and each collision event, a deterministic estimation is made of the fluence contribution at the detector point which is also shown in Figure 4. Since MCNP-X has special material definition process, the user has to consider the elemental composition of concrete and mass fractions on the base of their chemical composition and weight rates in definition of material in MCNP-X simulation. The percentages by weight of the different elements for different types of concrete are also given in Table 2.

\section{Results}

By considering the concrete sample which is defined in MCNP-X code, mass attenuation coefficient was calculated in range of $59.5 \mathrm{keV}, 80.9 \mathrm{keV}, 140.5 \mathrm{keV}, 356.5 \mathrm{keV}, 661.6 \mathrm{keV}$, $1173.2 \mathrm{keV}$, and $1332.5 \mathrm{keV}$ photon energies. To observe the transmissions of photons, different thicknesses of concrete sample were used. In this study, source has been considered as collimated isotropic source same as experimental conditions. To obtain accuracy of results, energy spectra at detector face quantified for each incident energy to determine amount of photon downscattering within the sample for each energy. In this study, a simple modeled geometry was used to estimate the transmission of photons through the modeled concrete sample with the different thicknesses.

Some comparison studies between Monte Carlo and XCOM data have been performed by Demir et al. by using FLUKA code. Table 3 shows the calculated mass attenuation coefficients of concrete sample and photon energies by giving calculated values by the XCOM [24] database and previously reported Monte Carlo values. Figure 5 shows the calculated mass attenuation coefficients of concrete sample by MCNPX. Deviations $\left(D=E_{a}-E_{b} / E_{b} \times 100 \%\right)$ between this study 
TABLE 3: Mass attenuation coefficients for the concrete sample.

\begin{tabular}{|c|c|c|c|c|}
\hline Energy (keV) & Present work (MCNP-X) & Demir et al. [13] & $\mathrm{XCOM}$ & Deviation $\left(D=E_{a}-E_{b} / E_{b} \times 100 \%\right)$ \\
\hline 59.5 & 0.205 & 0.203 & 0.207 & -0.97 to +0.97 \\
\hline 60.9 & 0.181 & 0.179 & 0.183 & -1.10 to 1.10 \\
\hline 140.5 & 0.148 & 0.144 & 0.154 & -2.70 to 4.05 \\
\hline 356.5 & 0.113 & 0.110 & 0.112 & -0.88 to -2.65 \\
\hline 661.6 & 0.085 & 0.082 & 0.086 & -3.52 to 1.17 \\
\hline 1173.2 & 0.066 & 0.064 & 0.066 & -3.03 to 0 \\
\hline 1332.5 & 0.059 & 0.058 & 0.061 & -1.69 to 3.38 \\
\hline
\end{tabular}

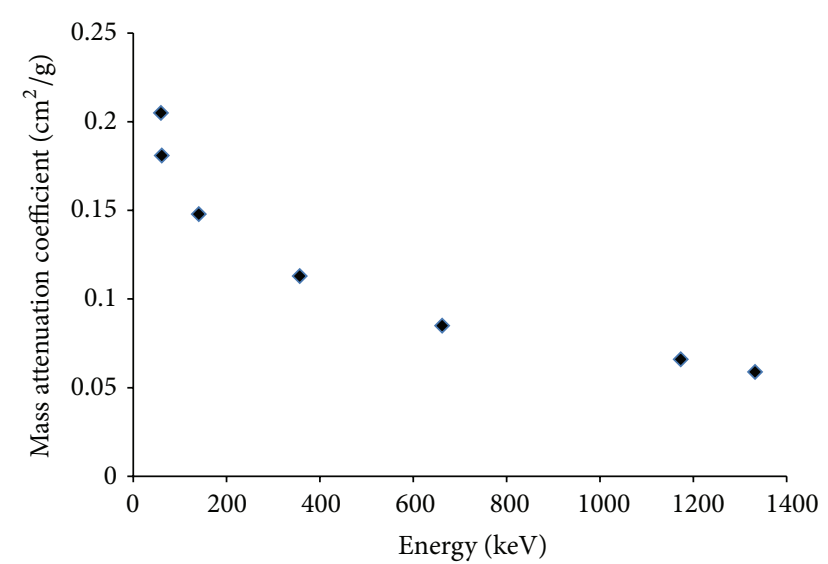

FIGURE 5: Calculated mass attenuation coefficients of concrete sample by MCNP-X.

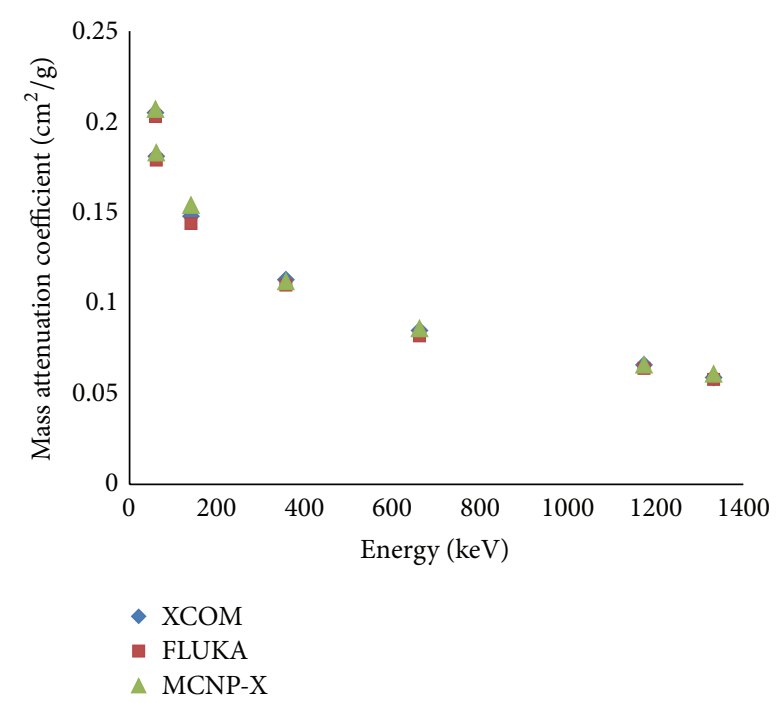

Figure 6: Comparison of mass attenuation coefficients of concrete sample.

and other results from Demir et al. and XCOM are given in Table 3.

The MCNP-X simulation code was employed to calculate the values of the mass attenuation coefficients for concrete sample. The MCNP-X versus XCOM and previously calculated values are plotted in Figure 6. It was found that the simulated results of mass attenuation coefficient values of the composites for seven gamma-ray energies were in good agreement with other data. Differences between the MCNP-X results of the XCOM and the MCNP mass attenuation coefficients could be due to deviations from narrowbeam geometry in the source-detector setup. Additionally, differences between the MCNP-X output results and FLUKA output results could be due to different cross section and data libraries of two different Monte Carlo codes. It has been found that the MCNP-X simulation and modeled $\mathrm{NaI}(\mathrm{Tl})$ detector can be applied to estimate the mass attenuation coefficients for various attenuator and energies in different future studies. In addition, it can also be concluded that the MCNP simulation code is a powerful method for evaluation of photon interaction parameters of the different types of materials.

\section{Conclusion}

In this study, as a validation of modeled detector, efficiency for different energies was obtained using MCNP-X code. In efficiency calculation of modeled $\mathrm{NaI}(\mathrm{Tl})$ detector, it was found that obtained results were moderately similar to available experimental data. Our MC model was capable of reproducing and confirming previous results on a commercial scintillation detector. Our study showed that MCNP-X code results are not only very similar to other experimental and Monte Carlo results in efficiency calculations but also very similar to other XCOM and FLUKA Monte Carlo results in mass attenuation coefficients calculations. Differences between the results in mass attenuation coefficient calculations could be due to different cross section data between MCNP-X and FLUKA and also could be due to computing time and statistical error rates. So the same geometry and model could be used for other applications such as attenuation studies with different compound materials as well as gamma spectroscopy for material characterization. As a conclusion topic, it can be also concluded that these results are useful in developing a better understanding of detector design by using Monte Carlo method and the Monte Carlo method is used in mass attenuation coefficients calculation. These results conclude that MCNP-X Monte Carlo simulation is in well compatibility with not only experimental data but also other Monte Carlo codes such as FLUKA code and can be applied to predict the mass attenuation coefficients for different attenuator and energies and can be an alternative method for experimental 
method since Monte Carlo has flexibility and convenience in defining geometry. Nowadays, radiation protection materials are currently in a rapid development by using new approaches such as doping complex materials into the concrete. It can be also concluded that modeled geometry can be used for future approaches such as new designs and new structures especially in the investigations of building materials which are used in the fields of radiation such as nuclear reactors and high energy radiation therapy facilities.

\section{Competing Interests}

The author declares that there are no competing interests regarding the publication of this paper.

\section{Acknowledgments}

The author would like to offer gratitude and special thanks to Mr. Baris Cavli (Regional Director of Affidea Turkey and Head of Turkish Society of Medical Radiotechnology TMRTDER).

\section{References}

[1] C.-Y. Yi and S.-H. Hah, "Monte Carlo calculation of response functions to gamma-ray point sources for a spherical $\mathrm{NaI}(\mathrm{Tl})$ detector," Applied Radiation and Isotopes, vol. 70, no. 9, pp. 21332136, 2012.

[2] S. Baccouche, D. Al-Azmi, N. Karunakara, and A. Trabelsi, "Application of the Monte Carlo method for the efficiency calibration of CsI and NaI detectors for gamma-ray measurements from terrestrial samples," Applied Radiation and Isotopes, vol. 70, no. 1, pp. 227-232, 2012.

[3] M. C. F. Moreira, C. C. Conti, and R. Schirru, "A new NaI(Tl) four-detector layout for field contamination assessment using artificial neural networks and the Monte Carlo method for system calibration," Nuclear Instruments and Methods in Physics Research Section: A, vol. 621, no. 1-3, pp. 302-309, 2010.

[4] S. H. Vegors Jr., L. L. Marsden, and R. L. Heath, "U.S. Atomic Energy Commission," (USAEC) Report 17 IDO-16370, 1958.

[5] W. F. Miller and W. J. Snow, "NaI and CSI efficiencies and photofractions for gamma-ray detection," Nucleonics, vol. 19, no. 11, p. $174,1961$.

[6] L. Zikovsky and B. Chah, "A computer program for calculating $\mathrm{Ge}(\mathrm{Li})$ detector counting efficiencies with large volume samples," Nuclear Instruments and Methods in Physics Research Section A: Accelerators, Spectrometers, Detectors and Associated Equipment, vol. 263, no. 2-3, pp. 483-486, 1988.

[7] Y. S. Selim, M. I. Abbas, and M. A. Fawzy, "Analytical calculation of the efficiencies of gamma scintillators. Part I: total efficiency for coaxial disk sources," Radiation Physics and Chemistry, vol. 53, no. 6, pp. 589-592, 1998.

[8] A. Jehouani, R. Ichaoui, and M. Boulkheir, "Study of the $\mathrm{NaI}(\mathrm{Tl})$ efficiency by Monte Carlo method," Applied Radiation and Isotopes, vol. 53, no. 4-5, pp. 887-891, 2000.

[9] M. I. Abbas, "Analytical formulae for well-type NaI (Tl) and HPGe detectors efficiency computation," Applied Radiation and Isotopes, vol. 55, no. 2, pp. 245-252, 2001.
[10] A. Gültekin, G. Kaynak, and O. Gürler, "Determination of full energy peak efficiency of HpGe detector from 59.5 to 1332.5 keV,' Indian Journal of Pure and Applied Physics, vol. 44, no. 4, pp. 281-286, 2006.

[11] S. Yalcin, O. Gurler, G. Kaynak, and O. Gundogdu, "Calculation of total counting efficiency of a $\mathrm{NaI}(\mathrm{Tl})$ detector by hybrid Monte-Carlo method for point and disk sources," Applied Radiation and Isotopes, vol. 65, no. 10, pp. 1179-1186, 2007.

[12] T. Nakamura, "Monte Carlo calculation of efficiencies and response functions of $\mathrm{NaI}(\mathrm{Tl})$ crystals for thick disk gammaray sources and its application to $\mathrm{Ge}(\mathrm{Li})$ detectors," Nuclear Instruments and Methods, vol. 105, no. 1, pp. 77-89, 1972.

[13] N. Demir, U. A. Tarim, M.-A. Popovici, Z. N. Demirci, O. Gurler, and I. Akkurt, "Investigation of mass attenuation coefficients of water, concrete and bakelite at different energies using the FLUKA Monte Carlo code," Journal of Radioanalytical and Nuclear Chemistry, vol. 298, no. 2, pp. 1303-1307, 2013.

[14] I. Akkurt, H. O. Tekin, and A. Mesbahi, "Calculation of detection efficiency for the gamma detector using MCNPX," Acta Physica Polonica A, vol. 128, no. 2, pp. 332-334, 2015.

[15] H. O. Tekin and U. Kara, "Monte Carlo simulation for distance and absorbed dose calculations in a PET-CT Facility by using MCNP-X," Journal of Communication and Computer, vol. 13, pp. 32-35, 2016.

[16] H.-X. Shi, B.-X. Chen, T.-Z. Li, and D. Yun, "Precise Monte Carlo simulation of gamma-ray response functions for an $\mathrm{NaI}(\mathrm{Tl})$ detector," Applied Radiation and Isotopes, vol. 57, no. 4, pp. 517-524, 2002.

[17] R. Casanovas, J. J. Morant, and M. Salvadó, "Energy and resolution calibration of $\mathrm{NaI}(\mathrm{Tl})$ and $\mathrm{LaBr} 3(\mathrm{Ce})$ scintillators and validation of an EGS5 Monte Carlo user code for efficiency calculations," Nuclear Instruments and Methods in Physics Research, Section A: Accelerators, Spectrometers, Detectors and Associated Equipment, vol. 675, pp. 78-83, 2012.

[18] M. A. Abdel-Rahman, E. A. Badawi, Y. L. Abdel-Hady, and N. Kamel, "Effect of sample thickness on the measured mass attenuation coefficients of some compounds and elements for 59.54, 661.6 and $1332.5 \mathrm{keV} \gamma$-rays," Nuclear Instruments and Methods in Physics Research, Section A: Accelerators, Spectrometers, Detectors and Associated Equipment, vol. 447, no. 3, pp. 432-436, 2000.

[19] I. Akkurt and A. M. El-Khayatt, "Effective atomic number and electron density of marble concrete," Journal of Radioanalytical and Nuclear Chemistry, vol. 295, no. 1, pp. 633-638, 2013.

[20] A. Akkaş, A. B. Tuğrul, O. Addemir, M. Marşoğlu, B. Ağacan, and B. Büyük, "Radiation shielding effect of boron carbide aluminum metal matrix composite," Acta Physica Polonica A, vol. 127, no. 4, pp. 947-949, 2015.

[21] M. E. Medhat, S. P. Shirmardi, and V. P. Singh, "Comparison of Geant 4, MCNP simulation codes of studying attenuation of gamma rays through biological materials with XCOM and experimental data," Journal of Applied \& Computational Mathematics, vol. 3, article 179, 2014.

[22] M. E. Medhat and V. P. Singh, "Geant4 Monte Carlo code application in photon interaction parameter of composite materials and comparison with XCOM and experimental data," Indian Journal of Pure and Applied Physics, vol. 54, no. 2, pp. 137-143, 2016.

[23] A. Ferrari, P. R. Sala, A. Fasso, and J. Ranft, "FLUKA: a multiparticle transport code," CERN-2005-010, INFN TC_05/11, SLAC-R-773, 2005. 
[24] M. J. Berger and J. H. Hubbell, NBSIR 87-3597: Photon Cross Sections on a Personal Computer, National Institute of Standards, Gaithersburg, Md, USA, 1987, http://physics.nist.gov/PhysRefData/Xcom/Text/XCOM.html. 

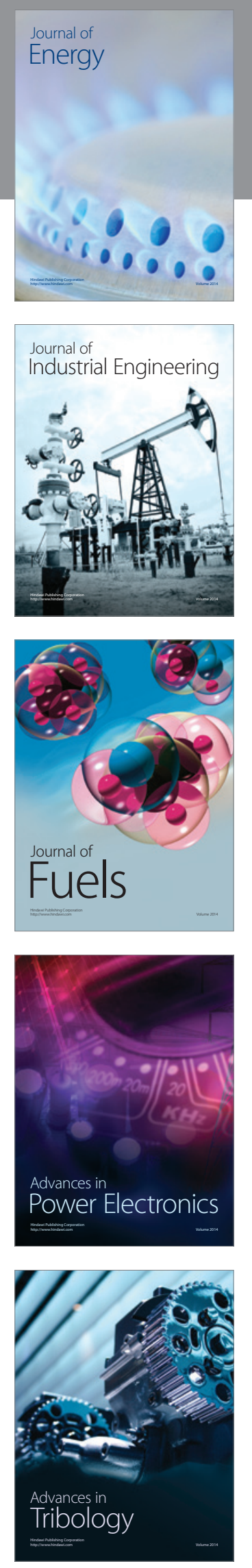
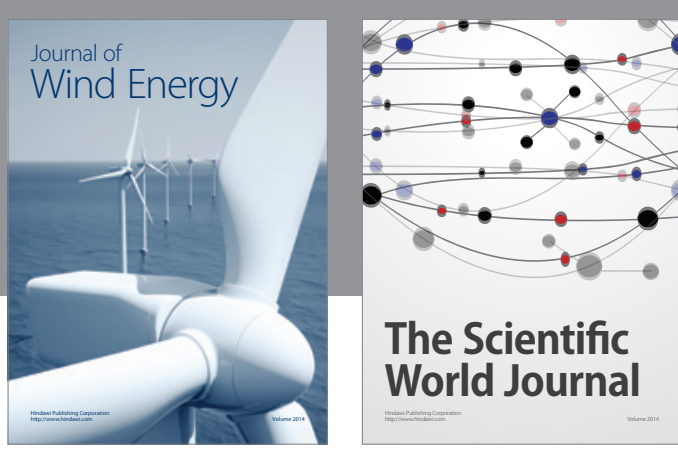

The Scientific World Journal
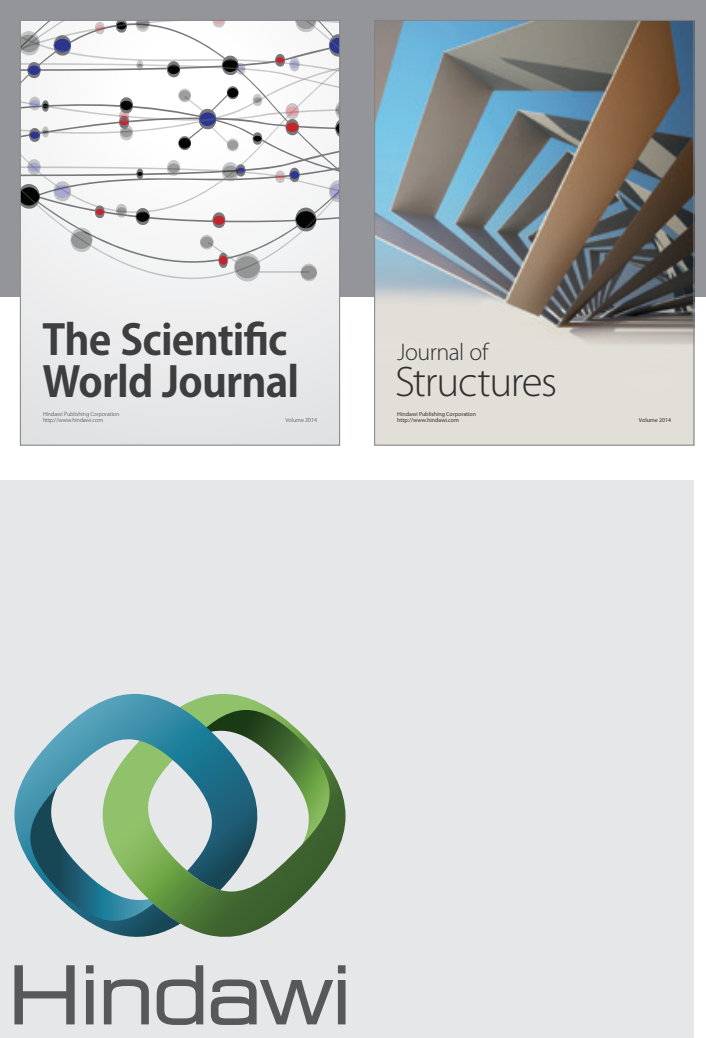

Submit your manuscripts at

http://www.hindawi.com
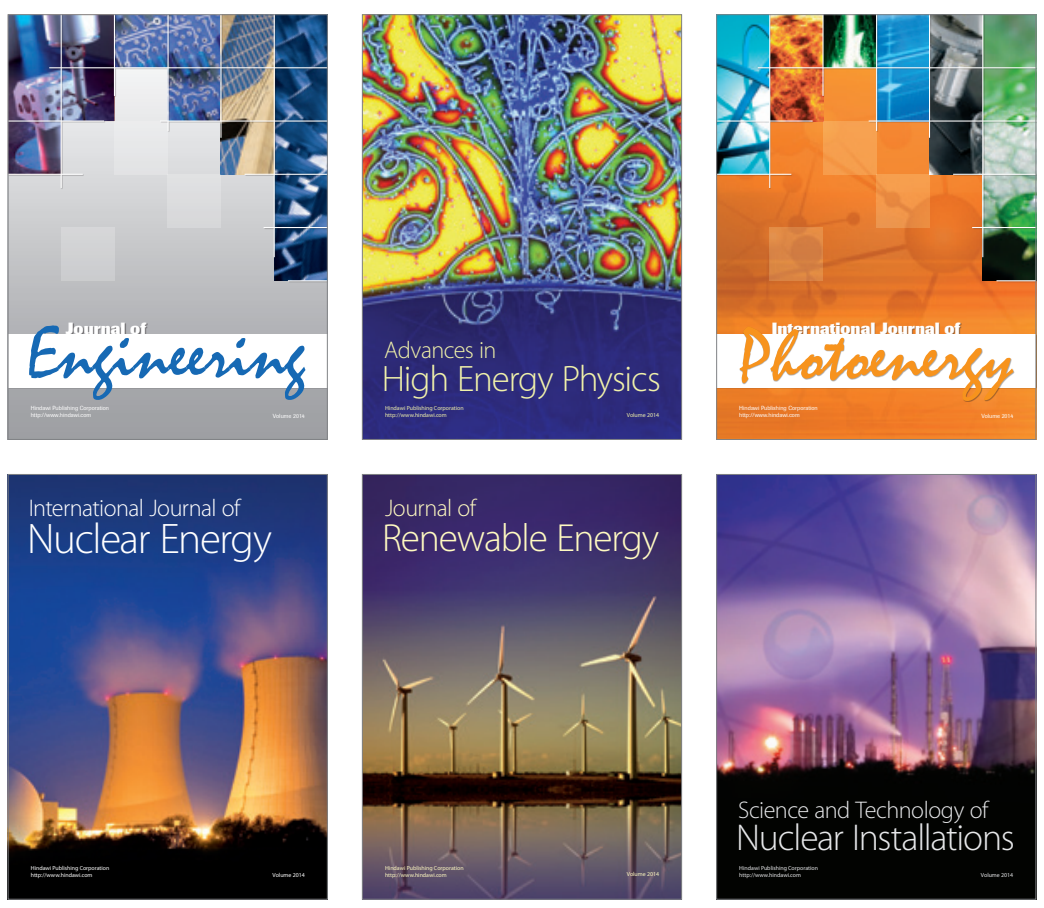
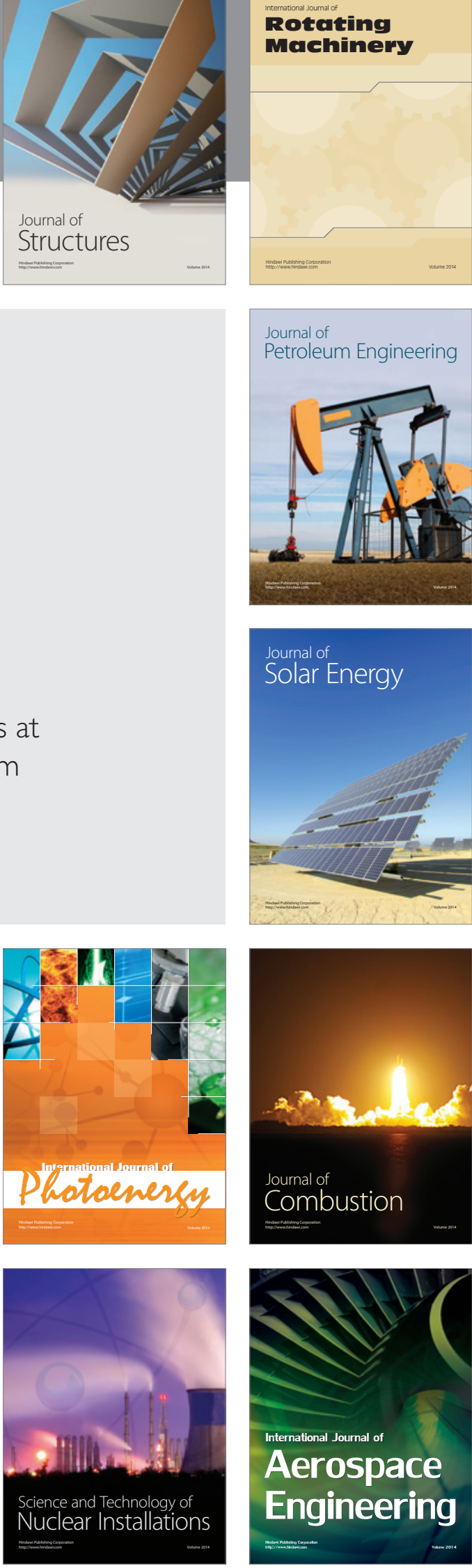